\title{
Investigation of heat transfer at flow of a gas in the quasitriangular channel with a constant temperature wall
}

\author{
Ekaterina Slesareva $^{1,{ }^{*}}$, Sergey Elistratov ${ }^{2}$, and Mihail Harisov ${ }^{2}$ \\ ${ }^{1}$ SB RAS, Kutateladze Institute of Thermophysics, 630090 Novosibirsk, Russia \\ ${ }^{2}$ Novosibirsk State Technical University, 630092 Novosibirsk, Russia
}

\begin{abstract}
The experimental research of heat exchange for gas flow in the quasitriangular channel has been carried out. Experiments have been executed for a case of a constant temperature of a wall. A thermogram of the temperature field on outlet of a channel by the thermal imaging camera was registered. The visualization of the temperatures field with the use of the mesh with small cells has been performed. It was shown, that the dependence between the changes of a friction coefficient from Reynolds number for the quasitriangular channel is similar to the known dependences for the smooth pipe; the average Nusselt number have monotone correlation from unitless parameter $\Psi$, and this correlation lies higher of the dependence for the round channel.
\end{abstract}

\section{Introduction}

In the presented work the heat exchange for air flow in the quasitriangular channel with a constant temperature of the wall was investigated. The panoramic thermographic method can be used for measurement of the temperature field at the channel outlet, as complementary to traditional methods [1-3]. In this method at visualization of a temperature field in a gas stream, the wire in a fine-mesh carries out the role of the indicator of temperature (fig. 1). The mesh parameters choose so that its influence on the field of velocity and temperatures in a gas flow was minimally. The thermograms has given possibility to see the panoramic picture of heating of gas in quasitriangular channel, and a full details of the evolution of the gas heating on the channel outlet.

\section{Measurement procedure}

In fig. 2 the scheme of a working section is shown. Walls of the quasitriangular channel 1 are made from the three strips of a copper foil in the thickness 50 micron and length $L=250 \mathrm{~mm}$. The channel form was set by means of inserts 4 in the form of circle segments. On an mounting disc 3 were fixed three the shaping circular segments so that internal curvature of walls of channel $R_{\mathrm{w}}$ was equaled $6.4 \mathrm{~mm}$. Hydraulic diameter of such

\footnotetext{
* Corresponding author: styuardessa@yandex.ru
} 
channel is $D_{\mathrm{h}}=1.31 \mathrm{~mm}$. The sealing of the channel cavity by the joints 8 was provided. For stabilization of a stream of air on the entrance of the channel into the input flange 5 the porous plate-membrane 6 has been installed. The constant temperature of wall $T_{\mathrm{w}}$ equal to $0^{\circ} \mathrm{C}$ by a thermal stabilization infill 7 (a mix of fine-grained particles of ice with water) was supported.
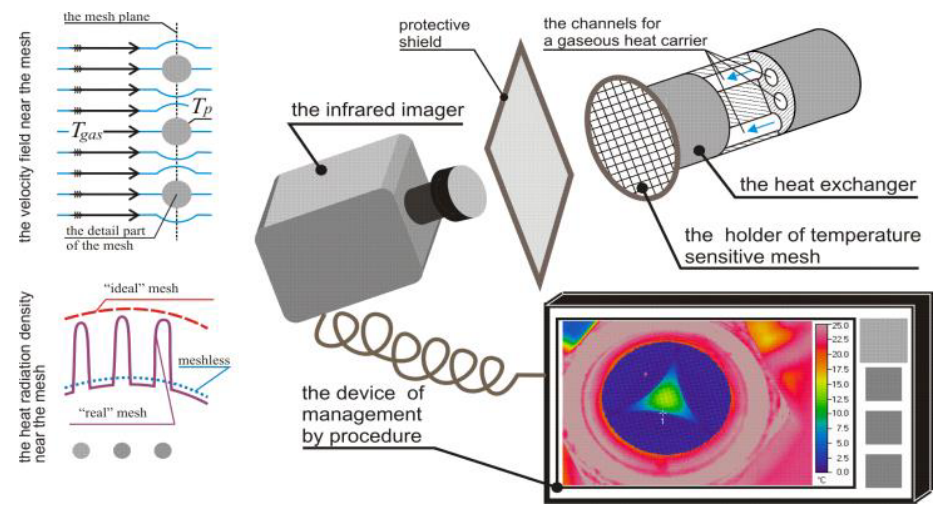

Fig. 1. Schematic picture of the panoramic thermographic method.

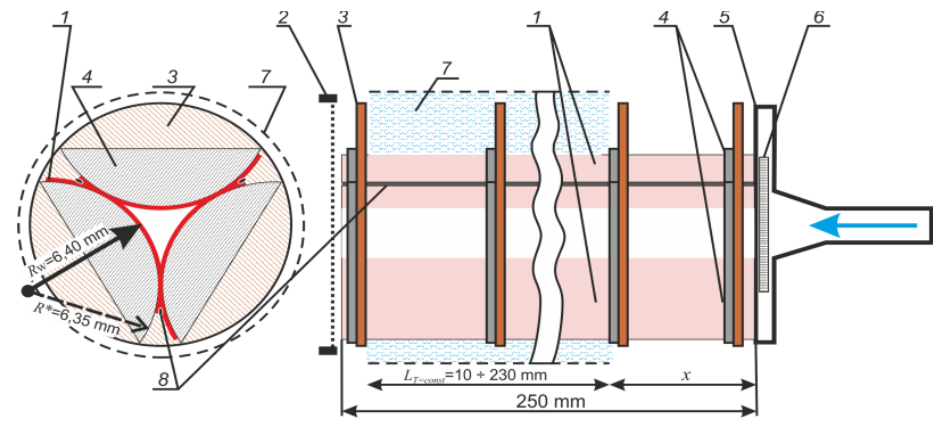

Fig. 2. Scheme picture of the test section: 1 - the wall of the quasitriangular channel, 2 - the holder of the mesh-thermal detector, 3 - mounting disc, 4 - the circular segment for a shaping, 5 - the input flange, 6 - the porous plate membrane, 7 - the infill for thermal stabilization , 8 - the sealing joint.

A during 200 $\div 800$ seconds, depending on value of flow rate and temperature air, the property of a thermal stabilization for the infill was maintained. The length of a section with constant temperature $L_{T=\text { const }}$ changed from 10 to $230 \mathrm{~mm}$, respectively the area of heat exchange surface $S$ of this section changed in range $200 \div 4600 \mathrm{Mm}^{2}$. Other part of the channel the kaolinite wadding thickness $10 \mathrm{~mm}$ was heat-insulated. The mesh-thermode, from a brass wire $80 \mu \mathrm{m}$ with a size of cell mesh $300 \times 300 \mathrm{~mm}$, was pressed to the outlet channel surface. Thermogram of the mesh-thermode by infrared imager NEC TH7100 (temperature accuracy $0.3 \mathrm{~K}$, spatial resolution $0.1 \mathrm{~mm}$, the exposure time $30 \mathrm{~ms}$ ) have been obtained. An air in the channel by the compressor ABN R Meko720 was injected. Before an air arrived into the compressor, it's through the dehumidifier with silica gel was passed. Flow rate by the needle valve on bypass line has regulated. The value of the flow rate air in test section by the flow meter Triton-gas-SGM 4 was measured. The average velocity air was calculated by formula:

$$
U=\frac{G}{\rho \cdot R_{\mathrm{w}}^{2} \cdot(\sqrt{3}-0.5 \cdot \pi)} .
$$

Static pressure on the channel entrance $P_{\text {inp }}$ and on the channel outlet $P_{\text {out }}$ by inspection 
manometers (a class of accuracy 0.15) has been measured. Measurements of a temperature of an air and a channel wall by the thermocouples accurate within $0.25 \mathrm{~K}$ have been measured. The average air temperature on the channel outlet by data from the thermograms has been calculated. The thermophysical properties of an air: $\rho-$ density, $v-$ viscosity, $C_{\mathrm{p}}$ - thermal capacity, $\lambda$ - heat conductivity, $\mathrm{Pr}-$ Prandtl number in depending from pressure $P$ and temperature $T$ were calculated based on the tabular data from the handbook $[4]$.

\section{Discussion of results}

The experimental research of heat transfer at gas flow in the quasitriangular channel with a constant temperature of the wall has been carried out. The experiment has been executed for the range of a air-mass flow $G$ from 0.06 to $0.6 \mathrm{~g} / \mathrm{s}$. Operation condition for experiments were transformed to an unitless parameters $(\mathrm{Re}, \mathrm{Pe}, \mathrm{Y})$ by formula:

$$
\operatorname{Re}=\frac{U \cdot D_{h}}{v} ; \operatorname{Pe}=\operatorname{Re} \cdot \operatorname{Pr} ; \Psi=\frac{1}{\operatorname{Pe}} \cdot \frac{L_{T=c o n s t}}{D_{h}}
$$

Used of experimental data of the static pressure on the entrance and on the outlet channel, the friction factor $\zeta$ for the case the air flow in the quasitriangular channel with a constant temperature wall has been obtained. The value of a friction factor was calculated by formula:

$$
\zeta=\left(P_{i n p}-P_{o u t}\right) \cdot \frac{D_{h}}{L} \frac{2}{\overline{\rho \cdot U^{2}}} .
$$

Figure 3 shows a change of the friction coefficient in dependence from the Reynolds number Re. As it is possible to see from graph the character of the friction coefficient change from a Reynolds number for the quasitriangular channel is similar to known dependences for a smooth pipe.

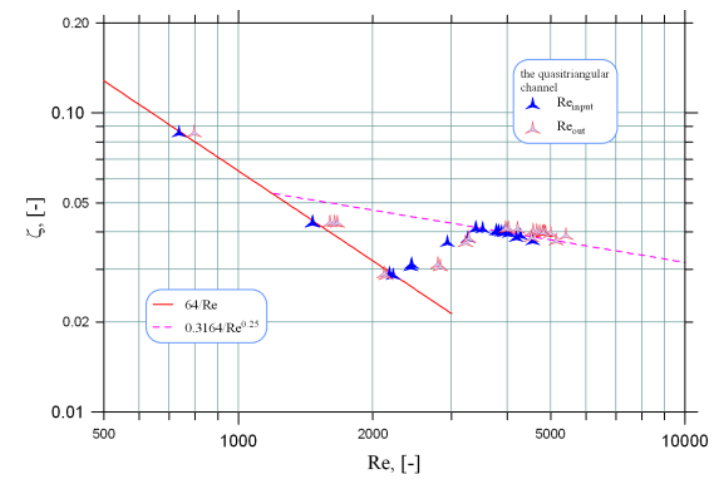

Fig. 3. The dependence between a change in friction coefficient $\zeta$ and Reynolds number Re for flow of the air in the quasitriangular channel with a constant temperature wall.

Using the data from the thermogram, the average Nusselt number $\overline{\mathrm{Nu}}$ for the area of heat exchange surface has been obtained. Calculation was performed by formula: $\overline{\mathrm{Nu}}=\frac{\mathrm{d} Q}{\mathrm{~d} t} \cdot \frac{1}{S \cdot\left(T_{w}-\bar{T}_{\text {air }}\right)} \cdot \frac{D_{h}}{\lambda} ; \bar{T}_{\text {air }}=\frac{\left[T_{T_{w}=\text { const }}+T_{\text {inp }}(x)\right]}{2} ; \frac{\mathrm{d} Q}{\mathrm{~d} t}=C_{p} \cdot G \cdot\left(T_{T_{w}=\text { const }}-T_{a d}\right)$, 
where $T(x)$ - the temperature air on the beginning heat exchange section, $x$ - the distance from the channel entrance to the beginning heat exchange section, $T_{\text {ad }}$ - the temperature air on the channel outlet with adiabatic wall on all length, $T_{T_{\mathrm{w}}=\mathrm{const}}$ - the temperature air on the channel outlet with constant temperature of the wall $T_{\mathrm{w}}$ on the section in length $H$. Figure 4 shows the dependence between the change the average Nusselt number and dimensionless parameter $\Psi$ for the quasitriangular channel with the constant temperature wall. You can see that Nusselt number have monotone correlation with the parameter $\Psi$. The dependence between Nusselt number and the parameter $\Psi$ has been interpolated by the fitted function: $\overline{\mathrm{Nu}}=3.51+\frac{0.15}{(\Psi+0.0001)^{0.85}}$. The dependence Petuhov [5] for the round channel is also shows in fig. 4. It is visible that the data for the quasitriangular channel lies higher of the dependence for the round channel.

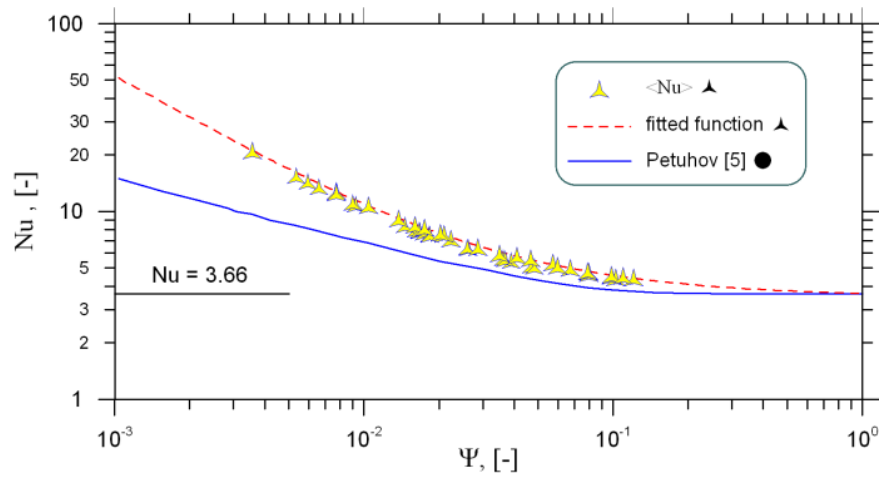

Fig. 4. The dependence between the change in average Nusselt number $\overline{\mathrm{Nu}}$ and dimensionless parameter $\Psi$ for the quasitriangular and circular channel with constant temperature wall.

\section{Conclusion}

The experimental research for flow of the air in the quasitriangular channel with constant temperature wall shown, that the dependence between the change in friction coefficient $\zeta$ and Reynolds number Re was consistent with known dependences in smooth pipes. It is shown, that the average Nusselt number have monotone correlation with unitless parameter $\Psi$, and data for the quasitriangular channel lies higher of the dependence for the round channel. It was shown, that the panoramic thermographic method can be used for the research of the heat exchange in the channel with any form of channels for the flow section at constant temperature of the wall.

This work was supported by the Russian Foundation for Basic Research, No. 16-38-00502-mol-a.

\section{References}

1. A.V. Efimova, A.V. Zaitsev, B.P. Zhilkin, D.N. Tokarev, K.V. Zaitsev, and Kh. Dashpuntsag, Bulletin of USTU: Thermal Engineering 33, 139 (2004)

2. S.L. Elistratov, E.Yu. Slesareva, EPJ Web of Conf. 82, 01060 (2015)

3. V.E. Nakoryakov, S.Y. Misyura, Energy and Fuels 27, 7089 (2013)

4. G.F. Hewitt Heat Exchanger Design Handbook. Part 5. Physical Properties (Hemisphere Publishing Corporation, Carlsbad, CA, 2002) 
5. B.S. Petuhov Heat exchange and resistance under a laminar flow fluid in tubes. (Energy, Moscow, 1967) 